Editorial: Hospital Metropolitano

ISSN (impreso) 1390-2989 - ISSN (electrónico) 2737-6303

Edición: Vol. 28 No 4 (2020) octubre - diciembre

DOI: https://doi.org/10.47464/MetroCiencia/vol28/4/2020/60-67

URL: https://revistametrociencia.com.ec/index.php/revista/article/view/97

Pág: $60-67$

\title{
Valoración del riesgo de caries dental en niños de 6 a 11 años de edad de la unidad educativa Jorge Escudero de la ciudad de Quito mediante el instrumento CAT
}

\author{
Assessment of dental caries risk in children from 6 to 11 \\ years of age of the Jorge Escudero educational unit of the \\ city of Quito through the CAT instrument
}

\section{Sandra Marlene Pérez Gallegos' ${ }^{(i)}$, María José Rodríguez Albuja ${ }^{2}$ iD}

\author{
Odontóloga por la Facultad de Odontología de la Universidad Central del Ecuador, Quito, Ecuador \\ Docente de la Facultad de Odontología de la Universidad Central del Ecuador, Quito, Ecuador ${ }^{2}$
}

Recibido: 05/10/2020 Aceptado: 15/10/2020 Públicado: 29/10/2020

\section{RESUMEN}

Objetivo: Valorar el riesgo de caries dental en niños de 6 a 11 años de edad de la Unidad Educativa Jorge Escudero de la ciudad de Quito mediante el instrumento CAT. Materiales y métodos: Estudio transversal realizado en 283 niños de 6 a 11 años de edad. Se evaluó el riesgo de caries mediante el instrumento validado CAT (Caries-risk Assessment Tool), el cual consta de 14 ítems, dividido en dos partes: un cuestionario dirigido a los padres de familia, complementándose con una exploración clínica oral al niño, previa estandarización de criterios con el fin de minimizar la variabilidad inter-examinador. Se realizó una prueba piloto en 20 personas adultas que no pertenezcan al tamaño de la muestra. Resultados: Se obtuvo un $98,2 \%$ de riesgo alto de caries y un $1,8 \%$ de riesgo moderado. No hubo diferencias significativas en la edad del niño donde $(p=0,057)$ fue superior a 0,05 , sin embargo en el sexo femenino frente al masculino hubo diferencia significativa de $(p=0,024)$. El nivel socioeconómico está directamente relacionado con el riesgo alto de caries, donde ( $p$ = 0,002). Conclusión: Se evidenció que los principales factores de riesgo de caries dental son la presencia de placa bacteriana visible y la presencia de zonas de esmalte desmineralizadas. Además existe una correlación pequeña o casi nula en lo que refiere a lo indicado por parte de los padres de familia en la encuesta y lo observado durante el examen clínico del niño con respecto al riego de caries.

Palabras claves: Valoración; riesgo; caries dental; CAT.

\section{ABSTRACT}

Objective: To assess the dental caries risk in children from 6 to 11 years of age of the Jorge Escudero Educational Unit of the city of Quito through the CAT instrument. Materials and methods: Cross-sectional study conducted on 283 children aged 6 to 11 years old. The caries risk was assessed using the validated CAT (Caries-risk Assessment Tool), which consists of 14 items, divided into two parts: a questionnaire addressed to the parents, complemented by an oral clinical examination of the child, prior standardization of criteria in order to minimize inter-examiner variability. A pilot test was performed on 20 adults who do not belong to the sample size. Results: There was a $98.2 \%$ high risk of caries and a $1.8 \%$ moderate risk. There were no significant differences in the age of the child where $(p=0.057)$ was greater than 0.05 ; however, in female versus male there was a significant difference of $(p=0.024)$. The socioeconomic level is directly related to the high risk of caries, where $(p=0.002)$. Conclusion: It was evidenced that the main risk factors for dental caries are the presence of visible bacterial plaque and the presence of demineralized enamel areas. In addition, there is a small or almost zero correlation regarding what is indicated by the parents in the survey and what was observed during the clinical examination of the child regarding the caries risk.

Keywords: Assessment; risk; dental caries; CAT.

\section{IDs Orcid}

https://orcid.org/0000-0002-6125-6961 https://orcid.org/0000-0002-5656-7781

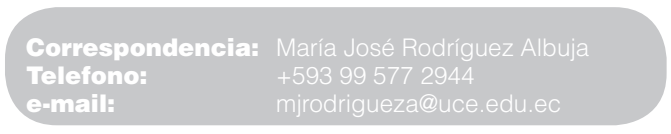




\section{INTRODUCCIÓN}

La caries dental es una enfermedad crónica multifactorial que afecta a todas las edades, pero más comúnmente se presenta en la infancia ${ }^{1}$. Según la Organización Mundial de la Salud (OMS) entre el $60 \%$ y $90 \%$ de la población de escolares en todo el mundo presenta caries, por lo tanto sigue considerándose un problema de salud pública².

La Federación Dental Internacional de Brasil en el 2015 estableció que la caries dental es la condición más prevalente que afecta a un 44\% de la población mundial y esta se debe a la continua y abundante exposición de azúcares libres y otros factores de riesgo como el nivel socioeconómico familiar, deficientes hábitos de higiene oral y falta de cuidados orales adecuados que juegan un papel importante en la salud oral ${ }^{3}$.

El Cuestionario Caries-risk Assessment Tool (CAT) propuesto por la Sociedad Americana de Odontopediatría es un método práctico, fácil y efectivo al ser aplicado por el examinador, que determina los principales factores de riesgo cariogénico y los indicadores de salud oral que presenta el niño, la aplicación de este instrumento es una contribución importante ya que gracias a este estudio se obtendrá estadísticas en relación al riesgo de caries en la población ecuatoriana, con el propósito de disminuir la prevalencia de caries y contribuir con información para futuras investigaciones que se realicen en el país.

Es consecuente hablar acerca de la importancia que tiene la valoración de riesgo de caries, que es un proceso clínico adecuado y oportuno para establecer la probabilidad individual de un paciente a desarrollar lesiones de caries en un futuro, o a su vez lesiones incipientes puedan producir una destrucción de tejido en un tiempo determinado, gracias a la identificación del rol que tienen varios factores asociados en el inicio y progreso de las lesiones dentales, la aplicación de instrumentos para la valoración de riesgo de caries dental, permitirá desarrollar actividades de prevención y un mejor control de la enfermedad en cada caso en particular.

La valoración del riesgo de caries dental en niños 6 a 11 años de edad en la Unidad Educativa Jorge Escudero a través del instrumento CAT y asignar el tipo de riesgo que presenta la población, permite establecer recomendaciones con respecto a la necesidad de implementación y divulgación de mecanismos e instrumentos que faciliten el diagnostico de riesgo de caries, de no realizarlo de manera adecuada se puede considerar un tratamiento incompleto, ya que la restauración de lesiones cariosas no cura la enfermedad, debido a que no previenen la aparición de futuras lesiones ${ }^{1}$.

\section{MATERIALES Y MÉTODOS}

Esta investigación fue aprobada por el Sub-comité de Ética de Investigación de la Universidad Central del Ecuador (CEISH-UCE), se realizó un muestreo probabilístico de tipo aleatorio simple, es un estudio de tipo observacional analítico y transversal, para el cálculo de la muestra se aplicó la fórmula de población finita obteniendo como resultado 283 niños y niñas entre 6 a 11 años de edad que acuden regularmente a la Unidad Educativa Jorge Escudero.

Los criterios de selección fueron: niños y niñas de 6 a 11 años de edad legalmente matriculados en la en la Unidad Educativa Jorge Escudero, que sus representantes legales aceptaron la participación y firma del consentimiento informado, además mostraron colaboración durante el exámen clínico. Los padres de los niños que no saben leer ni escribir y los niños que no asistieron el día de la evaluación clínica a la unidad educativa no fueron tomados en cuenta para el estudio.

\section{Prueba Piloto}

Se realizó una prueba piloto en 20 personas adultas que no pertenecían al tamaño de la muestra con el objetivo de identificar la semántica y la comprensión de las preguntas y términos del cuestionario, de la misma manera se determinó el tiempo de resolución del mismo que es de aproximadamente 10 minutos.

\section{Estandarización}

Fue necesario realizar una estandarización para que los datos sean fiables y evitar sesgos en la investigación, esta comprendió en capacitaciones teóricas y prácticas, el examen clínico fue realizado por dos examinadores aplicando los siguientes índices: Índice de Higiene Oral Simplificado (IHOS), Índice Gingival de LÖE y SILNESS, Índice del Sistema Internacional para la Detección y Evaluación de Caries (ICDAS II), Índice de DEAN para Fluorosis dental e Índice de la Sociedad Europea de Odontopediatría para el diagnóstico de Hipomineralización Inciso Molar; se aplicó el coeficiente del test de Kappa Cohen con la finalidad de obtener una concordancia inter-observadores, obteniendo una correlación equivalente a Muy Buena entre Docente - Investigador.

\section{Cuestionario}

Una vez obtenida la firma del consentimiento informado de cada uno de los 283 estudiantes que forman parte de la muestra, se procedió a la aplicación del Instrumento validado CAT (Caries-risk Assessment Tool) (Anexo 1) para la valoración de riesgo de caries dental en los niños y niñas de 6 a 11 años de edad, el cual consta de dos partes: la primera parte del instrumento se aplicó a los padres de familia quienes proporcionaron datos como institución en la 
que estudian, nombre, edad, sexo, curso en el que se encuentran y además respondieron 10 preguntas correspondientes a los factores de riesgo presentes en sus representados.

\section{Examen clínico}

Una vez obtenidos los datos sociodemográficos, se procedió a la evaluación clínica en la cavidad oral de los niños y niñas observando manifestaciones clínicas presentes en su boca, se ubicó al paciente en un lugar cómodo, con la presencia de luz natural y mediante una fronto luz, y se solicitó que se retire cualquier aparato de ortopedia que se encuentre en la cavidad oral, procedió a realizar el examen clínico utilizando un espejo bucal NO 5 y la sonda OMS, para observar la presencia o ausencia de placa dental siguiendo los criterios del Índice de Higiene Oral simplificado (IHOS).

Se realizó la inspección de la cavidad bucal para observar la presencia o ausencia de Gingivitis mediante el uso de un espejo bucal NO 5, la sonda OMS y la aplicación de aire en el tejido gingival siguiendo los criterios de Índice Gingival de LÖE y SILNESS.

Posteriormente se entregó al paciente una pasta y un cepillo dental para que se cepille los dientes con el objetivo de eliminar la placa bacteriana de las piezas dentales y obtener un mejor examen visual, una vez limpios los dientes del paciente, se observó la presencia o ausencia de mancha blanca mediante el examen visual en húmedo, para posteriormente secar la superficie del diente por 5 segundos con la jeringa triple dental y así realizar el examen visual en seco, con la ayuda de un espejo bucal NO 5 y una sonda OMS, siguiendo los criterios del Índice del Sistema Internacional para la Detección y Evaluación de Caries (ICDAS II)

Finalmente se realizó la inspección visual para observar la presencia o ausencia de Defectos de Esmalte siguiendo los criterios dados por Índice de DEAN para Fluorosis dental y el Índice de la Socie- dad Europea de Odontopediatría para el diagnóstico de Hipomineralización Inciso Molar.

\section{Análisis Estadístico}

Los datos obtenidos fueron tabulados en Microsoft Excel 2010 \& y posteriormente se ingresó en el programa SPSS V.22 de IBM, mismos datos descriptivos que se presentan mediante tablas y gráficos, aplicándose también la prueba de normalidad y las pruebas no paramétricas de Chi cuadrado, con un nivel de significancia del 5\% $(p<0.05)$.

\section{RESULTADOS}

De un total de 283 niños evaluados en forma general se determinó que el 1,8\% tienen un mediano riesgo de caries dental mientras que el $98,2 \%$ tienen un alto riesgo de caries, que representa el valor más alto (tabla 1).

Tabla 1. Nivel de riesgo de caries mediante CAT

\begin{tabular}{lcc}
$\begin{array}{c}\text { Riesgo de caries } \\
\text { mediante CAT }\end{array}$ & Frecuencia & Porcentaje \\
\hline Mediano riesgo & 5 & $1,8 \%$ \\
Alto riesgo & 278 & $98,2 \%$ \\
Total & 283 & $100,0 \%$ \\
\hline
\end{tabular}

Fuente: Sandra Pérez Gallegos

La relación del riesgo de caries dental mediante el instrumento CAT con respecto a la edad del niño, determinado en la prueba Chi cuadrado de Pearson presenta un valor del nivel de significación ( $p=$ 0,057 ) lo cual determina que no existen diferencias significativas (tabla 2), al contrario de lo observado en cuestión a la relación del riesgo de caries dental con el sexo del niño, en la prueba Chi cuadrado de Pearson el valor del nivel de significación fue ( $p=$ 0,024 ), los porcentajes entre masculino y femenino no son similares con relación a riesgo lo que determina que hubo diferencias significativas (tabla 3).

Tabla 2. Tablas cruzadas: Edad niño *Riesgo de caries mediante CAT

\begin{tabular}{|c|c|c|c|c|c|c|c|c|c|c|c|c|c|c|c|}
\hline \multicolumn{16}{|c|}{ Edad niño } \\
\hline \multirow{2}{*}{$\begin{array}{c}\text { Riesgo de } \\
\text { caries } \\
\text { mediante } \\
\text { CAT }\end{array}$} & \multicolumn{2}{|c|}{6 años } & \multicolumn{2}{|c|}{7 años } & \multicolumn{2}{|c|}{8 años } & \multicolumn{2}{|c|}{9 años } & \multicolumn{2}{|c|}{10 años } & \multicolumn{2}{|c|}{11 años } & \multicolumn{2}{|c|}{ Total } & \multirow{2}{*}{$p=$} \\
\hline & Cant & $\%$ & Cant & $\%$ & Cant & $\%$ & Cant & $\%$ & Cant & $\%$ & Cant & $\%$ & Cant & $\%$ & \\
\hline $\begin{array}{l}\text { Riesgo } \\
\text { moderado }\end{array}$ & 3 & $6,3 \%$ & 0 & $0,0 \%$ & 0 & $0,0 \%$ & 0 & $0,0 \%$ & 0 & $0,0 \%$ & 2 & $4,3 \%$ & 5 & $1,8 \%$ & \\
\hline $\begin{array}{l}\text { Alto } \\
\text { riesgo }\end{array}$ & 45 & $93,8 \%$ & 47 & $100,0 \%$ & 47 & $100,0 \%$ & 48 & $100,0 \%$ & 47 & $100,0 \%$ & 44 & $95,7 \%$ & 278 & $98,2 \%$ & 0,057 \\
\hline Total & 48 & $100,0 \%$ & 47 & $100,0 \%$ & 47 & $100,0 \%$ & 48 & $100,0 \%$ & 47 & $100,0 \%$ & 46 & $100,0 \%$ & 283 & $100,0 \%$ & \\
\hline
\end{tabular}

Fuente: Sandra Pérez Gallegos 
Tabla 3. Tablas cruzadas: Sexo niño *Riesgo de caries mediante CAT

\begin{tabular}{|c|c|c|c|c|c|c|c|}
\hline \multicolumn{8}{|c|}{ Sexo niño } \\
\hline \multirow{2}{*}{$\begin{array}{l}\text { Riesgo de } \\
\text { caries } \\
\text { mediante } \\
\text { CAT }\end{array}$} & \multicolumn{2}{|c|}{ Masculino } & \multicolumn{2}{|c|}{ Femenino } & \multicolumn{2}{|c|}{ Total } & \multirow{2}{*}{$p=$} \\
\hline & Cant & $\%$ & Cant & $\%$ & Cant & $\%$ & \\
\hline $\begin{array}{l}\text { Riesgo } \\
\text { moderado }\end{array}$ & 5 & $3,5 \%$ & 0 & $0,0 \%$ & 5 & $1,8 \%$ & \multirow{3}{*}{0,024} \\
\hline Alto riesgo & 136 & $96,5 \%$ & 142 & $100,0 \%$ & 278 & $98,2 \%$ & \\
\hline Total & 141 & $100,0 \%$ & 142 & $100,0 \%$ & 283 & $100,0 \%$ & \\
\hline
\end{tabular}

Fuente: Sandra Pérez Gallegos

En relación al riesgo de caries dental con el nivel socioeconómico familiar del niño, la prueba Chi cuadrado de Pearson determinó que el valor del nivel de significación fue $(p=0,002)$ determinando que si hubo diferencias significativas (tabla 4).

Tabla 4. Tablas cruzadas: P7: Nivel Socioeconómico familiar *Riesgo de caries mediante CAT

\begin{tabular}{|c|c|c|c|c|c|c|c|c|c|}
\hline \multicolumn{10}{|c|}{ P7: Nivel Socioeconómico familiar } \\
\hline \multirow{2}{*}{$\begin{array}{c}\text { Riesgo de } \\
\text { caries } \\
\text { mediante } \\
\text { CAT }\end{array}$} & \multicolumn{2}{|c|}{ Bajo } & \multicolumn{2}{|c|}{ Medio } & \multicolumn{2}{|c|}{ Alto } & \multicolumn{2}{|c|}{ Total } & \multirow{2}{*}{$p=$} \\
\hline & Cant & $\%$ & Cant & $\%$ & Cant & $\%$ & Cant & $\%$ & \\
\hline $\begin{array}{l}\text { Riesgo } \\
\text { moderado }\end{array}$ & 0 & $0,0 \%$ & 5 & $6,2 \%$ & 0 & $0,0 \%$ & 5 & $1,8 \%$ & \multirow{3}{*}{0,002} \\
\hline Alto riesgo & 198 & $100,0 \%$ & 76 & $93,8 \%$ & 0 & $100,0 \%$ & 278 & $98,2 \%$ & \\
\hline Total & 202 & $100,0 \%$ & 81 & $100,0 \%$ & $100,0 \%$ & $100,0 \%$ & 283 & $100,0 \%$ & \\
\hline
\end{tabular}

\section{Fuente: Sandra Pérez Gallegos}

El número de factores correspondientes al nivel alto de riesgo de caries obtenidos en la entrevista a padres, el $2,5 \%$ de encuestados indican no tener ningún factor correspondiente a nivel alto de riesgo de caries, el 5,3\% indican tener un factor, el 22,3\% indican tener dos factores, el 33,2\% indican tener tres factores, el $28,6 \%$ indica tener cuatro factores y el $8,1 \%$ de los encuestados indican tener cinco factores (gráfico 1), mientras que el número de factores correspondientes al nivel alto de riesgo de caries obtenidos en el examen clínico realizado a los niños, el 1,8\% de los evaluados no presentan ningún factor correspondiente a nivel alto de riesgo de caries, el 1,4\% presentan un factor, el 64,3\% presentan dos factores, el 26,1\% presentan tres factores, el 6,4\% presentan cuatro factores (gráfico 2).

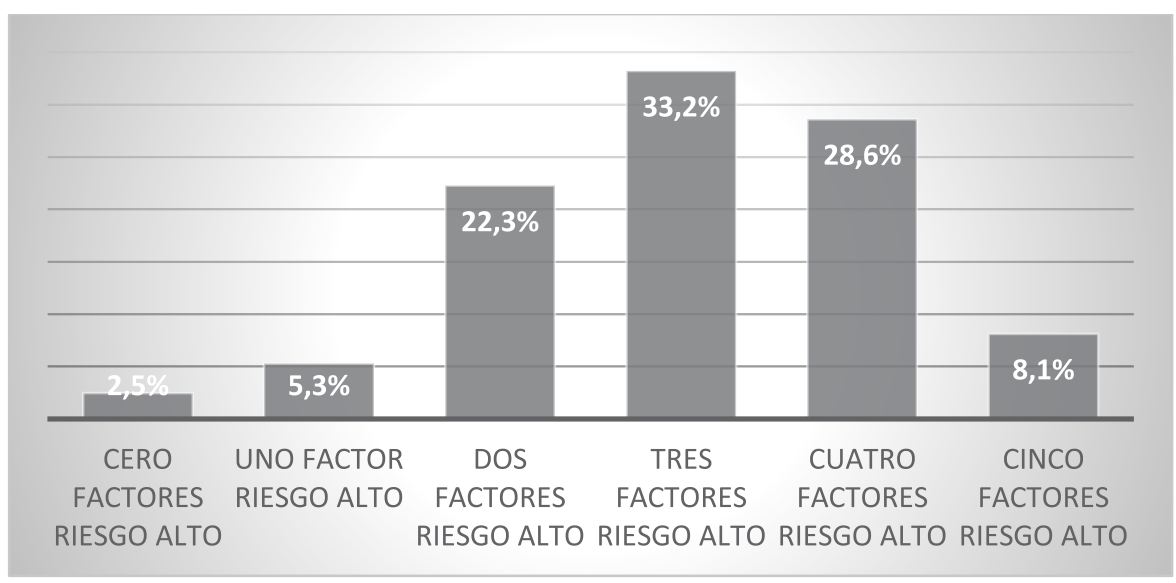

Gráfico 1. Número de factores correspondientes a riesgo alto obtenido de la entrevista a padres

Fuente: Sandra Pérez Gallegos 


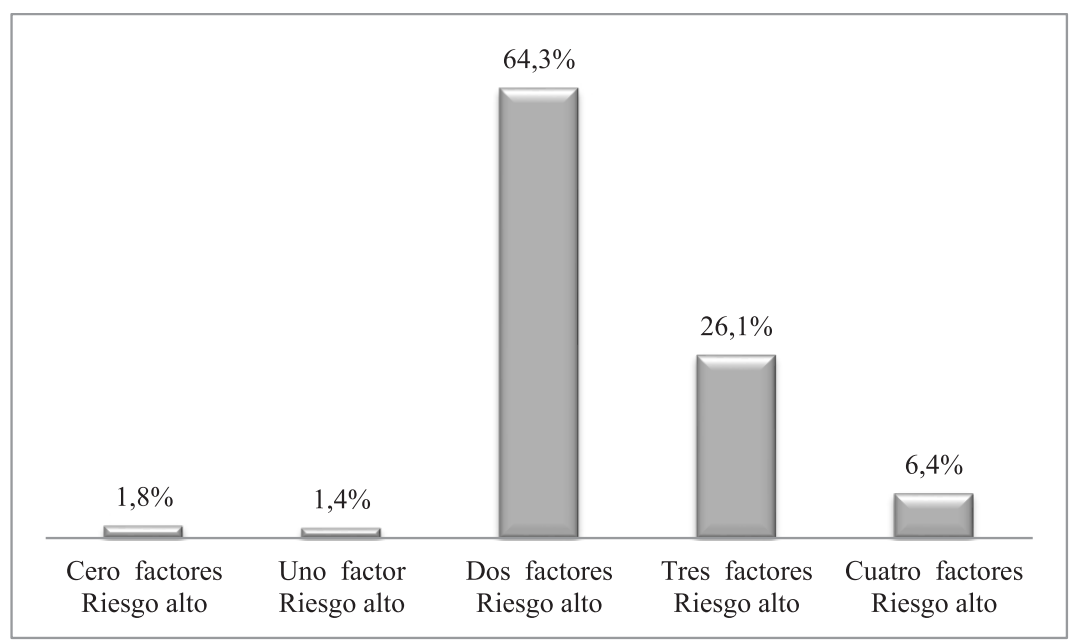

Gráfico 2. Número de factores correspondientes a riesgo alto obtenido del examen clínico

Fuente: Sandra Pérez Gallegos

El coeficiente de correlación de Sperman que es una medida no paramétrica de correlación de rango, determina que la relación entre la cantidad de ítems correspondientes a nivel alto de riesgo de caries obtenidos en la entrevista a padres (10 ítems totales) con el examen clínico realizado en los niños (4 ítems totales) es pequeña o casi nula ya que su resultado es 0,086 (tabla 5).

Tabla 5. Correlación entrevista de los padres y el examen clínico

\begin{tabular}{|c|c|c|c|}
\hline \multicolumn{4}{|c|}{ Correlaciones (Rho de Spearman) } \\
\hline \multirow{3}{*}{$\begin{array}{l}\text { Entrevistas } \\
\text { padres }\end{array}$} & & $\begin{array}{c}\text { Entrevistas } \\
\text { padres }\end{array}$ & $\begin{array}{l}\text { Examen } \\
\text { clínico }\end{array}$ \\
\hline & $\begin{array}{l}\text { Coeficiente de } \\
\text { correlación }\end{array}$ & 1 & 0,086 \\
\hline & Sig. (bilateral) & - & 0,149 \\
\hline \multirow[t]{2}{*}{ Examen clínico } & $\begin{array}{l}\text { Coeficiente de } \\
\text { correlación }\end{array}$ & 0,086 & 1 \\
\hline & Sig. (bilateral) & 0,149 & - \\
\hline
\end{tabular}

Fuente: Sandra Pérez Gallegos

\section{DIscusión}

Catalá, $2104^{9}$ afirma que la evaluación de riesgo de caries es de suma importancia dentro de la odontología, además de brindar servicios preventivos que conllevan a una ganancia económica y en salud oral, aseveraciones que coinciden con lo mencionado por Andrade y cols. $2014^{14}$ sobre la importancia de la evaluación del riesgo de caries, debido a que un pronóstico temprano, consiente en una planificación adecuada de tratamiento preventivo y curativo.

Estos resultados evidencian que se debe poner a prueba diferentes factores de riesgo en lo que corresponde a la caries dental, de esta manera se obtendrá el modelo de riesgo adecuado para futuras investigaciones, siendo los resultados de utilidad para tomar medidas de salud pública en la planeación de programas preventivos dirigidos a poblaciones vulnerables. Esta investigación y la utilización del instrumento CAT serían de gran aporte en la medida en que sea factible su aplicación en el desarrollo de programas preventivos tanto por parte del Ministerio de Salud Pública como a nivel de los consultorios privados dirigidos a la población ecuatoriana.

El presente estudio tuvo como objetivo valorar el riesgo de caries dental mediante el instrumento validado Caries risk Assessment Tool (CAT), en niños de 6 a 11 años de edad de la Unidad Educativa Jorge Escudero de ciudad de Quito, el cual fue propuesto por la Academia Americana de Odontología Pediátrica, dando como resultado un $98,2 \%$ para riesgo alto de caries y un 1,8 para riesgo moderado, datos que concuerdan a los descritos por Martínez C, Rafael et al. $2016^{18}$ en el estudio realizado en España, donde se consideró que todos los niños participantes superan el $94 \%$ de alto riesgo de caries infantil, de igual manera López et al $2018^{20}$ concluye en su investigación realizada en la Ciudad de México, encontrando un $75 \%$ que corresponde a un nivel de riesgo alto, un $23,8 \%$ para riesgo moderado y un $2,4 \%$ para riesgo bajo, determinando así que la etapa de la infancia es más vulnerable al riesgo alto de caries dental.

En esta investigación participaron 263 niños, el $49,8 \%$ correspondió al sexo masculino y el $50,2 \%$ al sexo femenino, es decir la distribución de la muestra fue equitativa en lo que se refiere a sexo, al igual que lo hizo López et.al $2018^{20}$ en su investigación tomando $50 \%$ de muestra correspondiente a sexo femenino y $50 \%$ al sexo masculino. Al realizar el análisis cruzado en nuestra investigación se obtuvo un $96,5 \%$ de riesgo alto y $3,5 \%$ para riesgo moderado en el sexo masculino, mientras que en el sexo femeino se obtuvo un $100 \%$ de riesgo alto. 
Con respecto a la edad se observó a los 6 años un $6,3 \%$ presenta un riesgo moderado y un $93,8 \%$ presenta un riesgo alto, de la misma manera se indica a los 11 años un 4,3\% presenta un riesgo moderado, y un $95,7 \%$ presenta riesgo alto, mientras que en las otras edades se encuentra un $100 \%$ de riesgo alto, lo que significa que no existe una relación relevante con respecto a la edad y el riego de caries, al igual que Casal $2014^{21}$ y Martínez C, Rafael et al. 2016 lo habían mencionaron, aseguran que existen otros factores que contribuyen a aparición de la enfermedad.

Una vez aplicado el instrumento CAT, se analizó las respuestas que se obtuvieron con mayor frecuencia, determinando así los factores de riesgo de caries más importantes, siendo la presencia de placa bacteriana visible y la presencia de zonas de esmalte desmineralizado, no dejando a un lado la relación que existe con el nivel socioeconómico familiar, ya que los evaluados pertenecientes al grupo de bajo nivel socioeconómico presentaron el 100\% de riesgo alto, lo que coincide con la investigación de Farsi $\mathrm{N}$, et al. $2013^{23}$, quien determinó que la desmineralización del esmalte y el estado socioeconómico son los factores de riesgo de caries más importantes, sin embargo difiere el estudio realizado por Córdova y cols $2016^{22}$, donde sugiere que los factores más significativos asociados a la caries dental fueron la perdida de piezas dentales en los padres y hermanos y el consumo de líquidos azucarado fuera de las comidas.

Se menciona además que de acuerdo a los resultados obtenidos, la relación con respecto al riesgo de caries obtenido en la entrevista a padres con el examen clínico realizado a los niños corresponde a valores de correlación de 0,086 la cual es pequeña o casi nula, es decir que lo mencionado por los padres acerca de la salud bucal de sus hijos, no corresponde en su totalidad con lo observado durante el examen clínico, indicando una falta de conocimiento en cuestión a la salud bucal de sus hijos.

\section{CONCLUSIONES}

El riesgo de caries dental se presentó en mayor proporción un riesgo alto y en menor proporción riesgo moderado. Los principales factores de riesgo de caries dental fueron la presencia de placa bacteriana visible y la presencia de zonas de esmalte desmineralizadas.

Se concluyó que no existe relación entre la edad del niño y el riesgo de caries, sin embargo se determinó que el sexo del paciente si tiene una relación con el riesgo de caries dental. El nivel socioeconómico está directamente relacionado con el alto riesgo de caries, debido a existe una mayor vulnerabilidad de los niños pertenecientes al grupo de nivel socioeconómico bajo.
De acuerdo a los datos obtenidos en la investigación, se evidenció que existe una correlación muy pequeña o casi nula en lo que refiere a lo indicado por parte de los padres de familia en la encuesta y lo observado durante el examen clínico del niño con respecto al riego de caries, demostrando que los padres de familia no conocen el estado de salud bucal de sus hijos.

\section{CONTRIBUCIÓN DE LOS AUTORES}

Sandra Marlene Pérez Gallegos: Concepción y diseño del trabajo; recolección y obtención de resultados; análisis e interpretación de datos; redacción del manuscrito.

María José Rodríguez Albuja: Concepción y diseño del trabajo; recolección y obtención de resultados; análisis e interpretación de datos; redacción del manuscrito.

\section{CONFLICTO DE INTERESES}

Los autores declararon no tener ningún conflicto de interés personal, financiero, intelectual, económico y de interés corporativo con el Hospital Metropolitano y los miembros de la revista MetroCiencia.

\section{FINANCIAMIENTO}

El financiamiento para la realización de la presente investigación fue financiado por los autores.

\section{REFERENCIAS BIBLIOGRÁFICAS}

1. Cerón-Bastidas XA. El sistema ICDAS como método complementario para el diagnóstico de caries dental. CES Odontol. 2015; 28 (2): 100-109.

2. Organización Mundial de la Salud. Encuestas de Salud Bucodental. Disponible en: http:// www.who.int/mediacentre/factsheets/ fs318/es/ (último acceso 12 octubre 2014).

3. Federación Dental Internacional (FDI). El Desafío de las Enfermedades Bucodentales. Una llamada a la acción global. [Online]; 2015 [cited 2018 Noviembre20.Disponibleen:https://www.fdiworlddental.org/ sites/default/files/media/documents/book_spreads_oh 2_spanish.pdf.

4. Cabeza G, González F, Paredes C. Estado de salud oral en el Ecuador. Revista OACTIVA UC. Cuenca. 2016; 3(1) 65-70, septiembre-diciembre. ISSN 24778915. Disponible en: http://oactiva.ucacue.edu. ec/index.php/oactiva/article/view/215/301.

5. Amado Schneider AR. Epidemiología de la caries dental en América latina Ecuador. Rev. De Odontopediatría Latinoamericana ALOP. 2014; 4.

6. Corrêa-Faria P, Paixão-Gonçalves S, Paiva S, Pordeus I. Incidence of dental caries in primary dentition and risk factors: a longitudinal study. Braz. Oral Res. 2016; 30(1):1-8.

7. Petersson G, Twetman S. Caries risk assessment in young adults: a 3 year validation of the Cariogram model. BMC Oral Health. 2015; 15:17.

8. Hallett KB. The application of caries risk assessment in minimum intervention dentistry. Aust Dent J. 2013; 58 Suppl 1:26-34.

9. Catalá Pizarro M, Cortés Lillo O. La caries dental: una enfermedad que se puede prevenir. An Pediatr Contin. 2014; 12(3): p. 147-151.

10. Mattos MA, Melgar RA. Riesgo de caries dental. Rev Estomatol Herediana 2004; 14(1-2): 101 - 106

11. Cárdenas $C$, Perona $M$. Factores de riesgo asociados a la prevalencia de caries de aparición temprana en niños de 1 a 3 años en una población peruana. Odontol Pediatr.2013 
12. Osorio J. Prevalencia de caries e índice COP en población escolar de 12 años del municipio de Copacabana 2013-2014. Rev. Estomatológica. 2015; 23(1):7-14

13. Bönecker, M. - Abanto, J. - Salete, M. - Pettorossi, J. - Guedes-Pinto, A. Problemas Bucales en Odontopediatría: Uniendo la Evidencia Científica a la Práctica Clínica. Madrid, España Ripano 2014.

14. Andrade PM, De la Cruz CD. Indicadores de prevalencia y de predicción de riesgo de caries dental. Rev Esp Cienc Salud 2014.

15. Bonecker M, Abanto J, Nahas M, Pettorossi J,Guedes A. Problemas Bucales en Odontopediatria: uniendo la evidencia Científica a la práctica clínica 2014; 63

16. Bettina Trinchitella A. Importancia de la salud oral y su conexión con la salud general. BIOMEDICINA. 2006; 2(3): p. 246-251.

17. Campos B. Nivel de riesgo de desarrollar caries dental en infantes de 0 a 3 años de edad. Maestría thesis, Universidad de San Carlos de Guatemala.S 2015.

18. Martinez C, Rafael et al. Valoración del riesgo de caries infantil en un servicio hospitalario de urgencias. Enferm. glob. [online]. 2016, vol.15, n.41, pp.1-9
19. Flores, Javier. (2001). Las bases biológicas de la diferenciación sexual humana en el siglo XXI. Desacatos, (8), 101-108. Recuperado en 12 de junio de 2019, Disponible en:http://www.scielo.org.mx/scielo. php?script=sci_arttext\&pid=S1607-050X2001000300007\&lng=es\&tIng $=$ es.

20. López OG, Linares VC, González GMB, Martínez GD, Morales ESL, Flores HND, Méndez OET. Nivel de riesgo e incidencia de caries en niños atendidos en clínica estomatológica de pregrado. Rev ADM 2018; 75.

21. Casals P. Guía de Práctica Clínica para la prevención y tratamiento no invasivo de la caries dental. RCOE. 2014;19(3):189-248

22. Córdova D, Flores $P$, Luna M, Tapia P, Prevalencia de caries dental y factores de riesgo en escolares de una institución educativa en Chiclayo, Perú.2016 KIRU. 2017;14(2):119 - 126. Disponible en: https:// doi.org/10.24265/kiru.2017.v14n2.03

23. Farsi N, Merdad L, Mirdad S. Caries risk assessment in preschool children in Saudi Arabia Caries. Oral Health Prev Dent. 2013;11(3):271. 80. doi: 10.3290/j.ohpd.a30479

24. Subsecretaría de Salud Pública. Guía clínica atencion primaria odontológica del preescolar de 2 a 5 años Santiago, Chile: Ministerio de Salud; 2009. 


\section{ANEXOS}

\section{EVALUACIÓN DE RIESGO DE CARIES ${ }^{24}$}

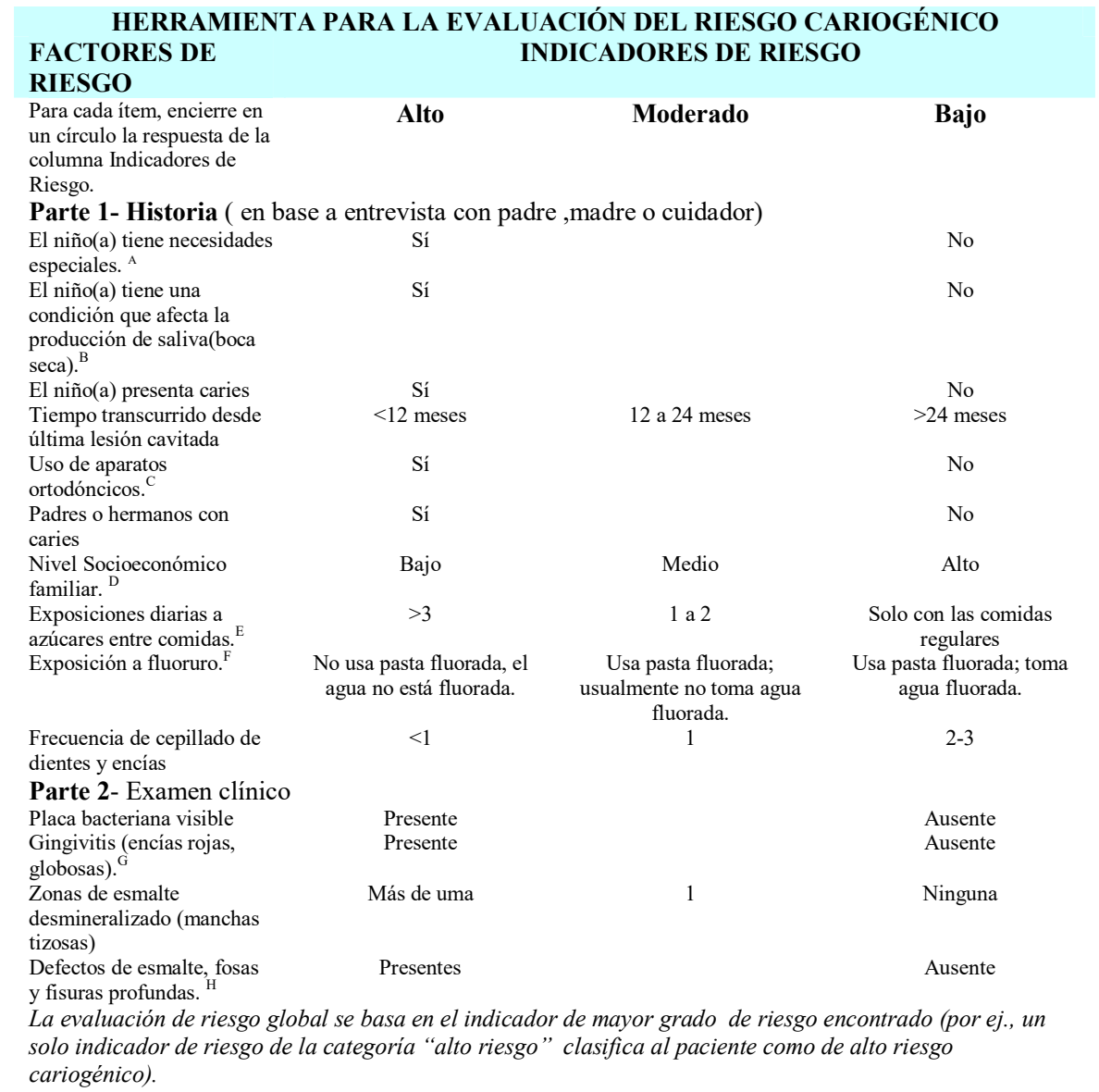

Leyendas de la tabla.

A) Niños(as) con necesidades especiales son aquellos que tienen una discapacidad física, del desarrollo, mental, sensorial, conductual, cognitiva o emocional o una condición limitante que requiere de manejo médico, una intervención de salud, y/o el uso de servicios especializados. La condición puede ser producto del desarrollo o adquirida, y puede causar limitaciones en el desarrollo de actividades de autocuidado de la vida diaria, o limitaciones substanciales en un área importante de la vida. El cuidado de la salud para pacientes con necesidades especiales está fuera de lo considerado rutinario y requiere de conocimiento especializado, mayor conciencia, atención e infraestructura.

B) La alteración en el flujo salival puede ser el resultado de condiciones congénitas o adquiridas, cirugía, radiación, medicación, o estar relacionada con la edad. Cualquier condición, tratamiento, o proceso conocido o reportado que altere el normal flujo salival debe considerarse como indicativo de riesgo, a menos que se demuestre lo contrario.

C) Los aparatos ortodóncicos incluyen tanto los fijos como los removibles, mantenedores de espacio, y otros dispositivos que se mantengan en boca por períodos prolongados, y que puedan retener restos de alimentos y placa, dificultar la higiene bucal, obstaculizar la exposición de las superficies dentarias a fluoruro, o crear un ambiente que favorezca la iniciación de caries.

D) Encuestas nacionales han demostrado que los niños y niñas que viven en hogares de ingresos medios y bajos tienen más probabilidad de presentar mayor prevalencia y severidad de caries, en relación a aquellos de hogares de mayores ingresos. Por esto, el nivel socioeconómico debe considerarse como un indicador inicial de riesgo, que puede ser compensado por la ausencia de otros indicadores de riesgo.

E) Ejemplos de fuentes de azúcares incluyen bebidas carbonatadas, galletas, queques, dulces, cereales, papas fritas envasadas, papas fritas, pretzels, pan, ketchup, jugos y frutas.

F) El uso no supervisado de pasta de dientes y el uso en el hogar de fluoruros tópicos no se recomiendan para niños sin la habilidad de expectorar bien desarrollada.

G) Aunque los microorganismos responsables de la gingivitis pueden diferir de aquellos implicados primariamente en la caries, la presencia de gingivitis es un indicador de pobre o infrecuente higiene bucal, y se ha asociado a la progresión de caries.

H) La anatomía dentaria y los defectos hipoplásicos pueden predisponer al niño o niña al desarrollo de caries. 